\title{
HPV Positive Oropharyngeal Cancer in Two Gynaecologists Exposed to Electrosurgical Smoke Plume
}

\author{
Jim Parker ${ }^{*}$ (iD) and Jonathan Clark ${ }^{2,3,4}$ \\ ${ }^{1}$ Obstetrician, Gynaecologist and Endoscopic Surgeon, Honorary Clinical Senior Lecturer, School of \\ Medicine, Faculty of Science, Medicine and Health, University of Wollongong, Australia \\ ${ }^{2}$ Department of Head and Neck Surgery, Sydney Head and Neck Cancer Institute, Chris O'Brien Lifehouse, \\ Australia \\ ${ }^{3}$ Royal Prince Alfred Institute of Academic Surgery, Sydney Local Health District, Australia \\ ${ }^{4}$ Faculty of Medicine and Health, Sydney Medical School, The University of Sydney, Australia
}

*Corresponding author: Dr. Jim Parker, BMed, BSC, DRANZCOG, FRANZCOG, Obstetrician, Gynaecologist and Endoscopic Surgeon, Honorary Clinical Senior Lecturer, School of Medicine, Faculty of Science, Medicine and Health, University of Wollongong, Australia

\begin{abstract}
Every day thousands of health-care professionals are exposed to surgical smoke plume produced by tissue destruction during electrosurgical procedures. There has been increased awareness of the possible health hazards associated with inhalation of a range of biological contaminants that have been identified in the aerosol created. This has corresponded to an increased number of observational and experimental studies aimed at identifying the specific risks to medical personnel and ways to control the levels of exposure to the variety of hazardous components that have now been identified. The chemical component of surgical smoke plume has been found to contain over 80 different toxic substances including physical (dust), chemical (known carcinogens, toxic gases, vapours, free radicals) and biological (blood, bacteria, viruses) material. A number of viruses have been identified in the respiratory aerosol including human immunodeficiency virus, hepatitis virus and human papillomavirus (HPV). A range of pathological conditions have been reported in health-care workers exposed to surgical smoke plume, including HPV positive oropharyngeal squamous cell carcinoma (OPSCC) in gynaecologists. The current two case reports support the existing evidence that electrosurgical smoke exposure during treatment for HPV positive lesions is a risk factor for the development of OPSCC, although a causal link has not been established.
\end{abstract}

\section{Case Presentations}

Patient 1 is a 64-year-old male gynaecologist who presented with a 3-month history of an asymptomatic right upper neck mass. Physical examination revealed a mobile non-tender neck mass measuring $3 \mathrm{~cm}$ in the anterior triangle of the right neck. A CT scan demonstrated a $3 \mathrm{~cm}$ soft-tissue lesion in the right upper neck. Nasopharyngeal endoscopy revealed a small mass on the inferior pole of the right tonsil. A fine needle aspiration biopsy (FNAB) of the neck mass showed a p16 positive squamous cell carcinoma (SCC). A PET scan showed enhancement in the right tonsil and a single right cervical lymph node. The cancer was staged as T2N1MO. He was treated with right neck dissection and a transoral robotic surgery (TORS) to remove the primary tumour. The histopathology confirmed squamous cell carcinoma.

Patient 1 had a 5 pack-year history of cigarette smoking 45 years ago and was a social alcohol drinker. He had been in a monogamous relationship for 31 years. He had performed approximately 250 electrosurgical procedures for HPV-related cervical dysplasia and vulvar lesions over a 27-year period. Most of the procedures were performed wearing a simple surgical face mask and without the use of a smoke evacuator. 
Patient 2 is a 66-year-old male gynaecologist who presented with an enlarged left upper jugular lymph node. Fine needle aspiration biopsy showed mild inflammation. Clinical follow-up showed no increase in size. Two years later symptoms of dysphagia developed and nasopharyngeal endoscopy revealed a $3 \mathrm{~cm}$ midline lesion of the tongue base. FNAB of the tongue showed a p16 positive SCC. Definitive treatment with concurrent chemoradiotherapy was given over a 7-week period.

Patient 2 had never smoked and was a social alcohol drinker. He had been in a monogamous relationship for 40 years. He had performed approximately 500 electrosurgical procedures for HPV-related cervical dysplasia and vulvar lesions over a 40-year period. Most of the procedures were performed wearing a simple surgical face mask and without the use of a smoke evacuator.

\section{Discussion}

Human Papilloma Virus is estimated to be a causative factor in greater than $5 \%$ of human cancers [1]. OPSCC is a subset of head and neck squamous cell cancers ( $\mathrm{HN}$ $\mathrm{SCC})$, representing approximately $1 \%$ of all cancers diagnosed globally each year [2]. OPSCC has two distinct aetiologies; traditionally the main risk factor has been tobacco and alcohol consumption, however over the last 20 years HPV infection has increasingly been recognized as the main causative agent [1]. This corresponds directly with a reduction in smoking rates in countries with effective anti-smoking campaigns, such as Australia. The proportion of HPV-related cancers now represents over 70 percent of all OPSCC cases and in some regions more than $90 \%$. HPV-16 and HPV-18 subtypes account for $70 \%$ of cervical cancers and HPV-16 accounts for approximately $95 \%$ of HPV-positive OPSCC [1,2].

Although the epidemiology of cervical HPV infection has been more extensively studied, HPV infection of theoropharynx has been found to have many similar characteristics and risk factors. Most HPV infections are asymptomatic and the majority of detected infections clear within two years [1,3]. Both OPSCC and cervical cancers have a long latency period of $10-30$ years and the principal risk factor for HPV-positive OPSCC and cervical cancer is sexual behaviour. Males are more likely to develop OPSCC and account for approximately $70 \%$ of cases. The specific anatomy of the tonsillar crypts, characteristics of the oropharyngeal microbiome, concurrent alcohol consumption, recurrent physical trauma to the oropharyngeal mucosa from eating, inhalation of airborne pollutants and exposure to multiple simultaneous carcinogens may also play a pathogenic role in susceptible individuals. In addition, a range of healthcare professionals, particularly gynaecologists, have the additional risk of inhalation of surgical smoke plume generated during electrosurgical procedures of highrisk HPV-related anogenital lesions $[3,4]$.

Gynaecologists regularly treat high-risk cervical in- traepithelial neoplasia (CIN) lesions, detected by PAP smear or HPV-DNA screening. Biopsy proven CIN 2/3 lesions are treated by removal of the cervical transformation zone using electrosurgical procedures including electrocautery, laser ablation and loop electrosurgical excision procedure (LEEP). Tissue destruction during electrosurgery results in the generation of significant amounts of gaseous by-product that is known as surgical smoke plume [5]. The aerosol created contains a large number of substances which have been found to be potential biohazards to surgeons and other healthcare workers. These include over 80 different toxic substances such as known carcinogens, toxic gases, vapours, free radicals, lung damaging particulates, intact cells, blood, bacteria and viruses $[5,6]$. A number of different viruses have been identified in the respiratory aerosol including human immunodeficiency virus, hepatitis virus, bovine papillomavirus and HPV. A large number of different HPV subtypes have been identified, including HPV-16, using polymerase chain reaction (PCR) techniques $[3,6,7]$.

Surgical smoke was officially recognised as a health hazard in 1985 when the US Centre for Disease Control and Prevention published a report stating that there is a "potential hazard from exposure to smoke generated by electrocautery (electrosurgery) knives" [8]. The potential health risks of surgical smoke include eye irritation, dermatitis, nausea, anaemia, infection, headaches, anxiety, oropharyngeal papilloma, carcinoma and multiple respiratory problems $[7,8]$. The presence of potentially carcinogenic by-products and HPV DNA in surgical smoke plume was first identified in 1989. Since this time, numerous studies have investigated the sequence of events that are required for transmission of HPV viral DNA from patient to gynaecologist during electrosurgery procedures [3,9]. Many studies have reported the presence of intact HPV DNA in smoke plume from HPV-positive lesions. HPV DNA has been consistently demonstrated in nasolabial, nasal and oral swabs of surgeons performing electrocautery [4].

Transmission of HPV-related disease was first reported in 1991 and there have been numerous case reports of human infection including laryngeal papillomatosis and OPSCC $[7,9]$. In particular, there are two previous case reports of gynaecologists who developed HPV p16 positive OPSCC after performing large numbers of LEEP procedures over 20 -30 years in gynaecological practice [9]. These case reports are remarkably similar to the two cases outlined in the present manuscript.

A recent large study of 700 gynaecologists from 67 hospitals in China took nasal swabs from surgeons performing LEEP procedures (469) compared with gynaecologists who did not perform LEEP (231) [3]. The prevalence of HPV DNA in the nasal swabs of surgeons who performed LEEP was $9.0 \%$ (42/469) compared to $1.7 \%(4 / 231)$ of participants who had not performed 
electrosurgery. The most common genotype detected was high-risk HPV 16 in 76\% (32/42) of the electrosurgery group. Participants who performed electrosurgery for more than 15 years were significantly more prone to HPV infection $(17 \%, 13 / 75)(P<0.001)$. During the 24-month follow-up, no cases of HPV-related OPSCC were detected but it should be noted that $83 \%$ of the 700 gynaecologists studied were female and the mean age was 35.1 years with only $6 \%$ over the age of 50 years. The authors concluded that gynaecologists who perform electrosurgical operations for cervical lesions, especially those exposed to repeated smoke plume over a long time, were at increased risk of HPV-DNA infection [3]. No studies have attempted to determine the incidence of OPSCC in older gynaecologists after many years of exposure to HPV-containing electrosurgical smoke plume.

A systematic review and meta-analysis of airborne HPV transmission risk identified 30 original studies reporting outcomes in medical staff performing ablation procedures [4]. Ten studies identified HPV DNA in ablation smoke from cautery procedures in aerosol samples, filters or air exhaust systems. Three studies found HPV genotypes in nasolabial and upper airway swabs of medical staff that corresponded to ablated patient lesions. One study found high-risk HPV genotypes in exhaust suction tubes that matched the resected CIN lesions. Bovine and murine models have documented HPV-related infection from HPV particles transmitted in cautery-generated smoke to animals. The authors of the systematic review concluded that simple safety measures greatly reduce HPV contamination and transmission risk [4]. It is also possible that HPV vaccination may provide some protection from infection and later development of OPSCC, although this has not yet been investigated.

A large number of studies have examined protective measures that may reduce transmission risk of known toxic components of surgical smoke plume from patients to health-care workers $[5,7]$. A comprehensive review of publications related to surgical masks concluded that basic masks provided partial protection because they only filtered out particulates above 5 micrometres $(\mu \mathrm{m})$. The particulate size of many of the identified contaminants such as bacteria $(0.3 \mu \mathrm{m})$ and viruses $(0.01$ to $0.3 \mu \mathrm{m}$ ) are much smaller. In addition, $77 \%$ of the particulate matter found in surgical plume is less than 1.1 $\mu \mathrm{m}$. The authors concluded that specialised high-filtration masks and filters are required in order to provide adequate protection but need to be used in conjunction with other measures. These include smoke evacuation systems with high efficiency filters, respirator filter systems and theatre air ventilation and filtration systems that increase air exchange rates $[7,10]$. Controlling inhaled respiratory aerosols has gained greater priority since it has been recognised that SARS-CoV-2 airborne transmission can occur $[6,10]$. Several international surgical societies have called for caution or even discourage the use of a laparoscopic approach during the current pandemic including the International Society of Gynecologic Endoscopy [6].

The health hazards of surgical smoke plume have been increasingly recognised by nursing and medical associations and regulatory bodies around the world $[6,8]$. The United States Occupational and Safety and Health Administration estimated that 500,000 theatre staff are exposed annually to electrosurgical smoke. These organisations recommend that perioperative staff take measures to minimise exposure risk, such as wearing surgical gloves and face masks and using smoke evacuation systems. Nevertheless, most surgeons and theatre staff are unaware of the potential hazards of smoke plume inhalation and the use of protective measures is often voluntary $[5,7]$.

Many countries (Scandinavia), states (NSW, Australia) professional organisations (United States Association of periOperative Registered Nurses) and regulatory bodies (United Kingdom Health and Safety Executive) have introduced guidelines for surgical plume devices used in clinical practice $[7,8]$. The recent SARS-CoV-2 pandemic is likely to expedite the more widespread introduction of similar policies $[6,10]$. Although this report has focused on the risks of smoke generated during electrocautery of anogenital HPV-related lesions, it is obvious that the health hazards of smoke produced during all surgical procedures warrants further investigation and regulation [6]. Gynaecologists should be informed about the dangers of surgical plume and ensure that all necessary measures are taken to protect operating room staff and patients.

\section{Conclusions}

Airborne dispersal of high-risk HPV DNA during electrosurgical ablation of HPV-associated lesions is well documented using PCR techniques. Recent large studies have demonstrated that the transmission rate from patient to gynaecologists is high (9\%) and increases with increasing years of exposure ( $>15$ years $=17 \%$ ). Matching high-risk genotypes in smoke plume and surgeon swabs have been found to correspond to the genotype of the tissue treated with electrocautery. All case reports of HPV-associated disease have been in healthcare workers treating anogenital lesions. The case reports of OPSCC in gynaecologists performing a high-volume of electrosurgical procedures for HPV-related anogenital lesions and available scientific literature, suggest that workplace exposure should be included as a risk factor for the development OPSCC. Efforts should be made to inform at risk health-care workers of the potential hazards of surgical smoke exposure and regulations introduced to ensure the implementation of mandatory protective measures. 


\section{Consent}

Both patients reported have given written consent to the de-identified presentation of their case histories.

\section{References}

1. Berman TA, Schiller JT (2017) Human Papillomavirus in cervical cancer and oropharyngeal cancer: One cause, two diseases. Cancer 123: 2219-2229.

2. (2018) Diet, nutrition, physical activity and cancer: A global perspective. A summary of the third ER.

3. Hu X, Zhou Q, Yu J, Wang J, Tu Q, et al. (2021) Prevalence of HPV infections in surgical smoke exposed gynecologists. Int Arch Occup Environ Health 94: 107-115.

4. Palma S, Gnambs T, Crevenna R, Jordakieva G (2021) Airborne human papillomavirus (HPV) transmission risk during ablation procedures: A systematic review and meta-analysis. Environ Res 192: 110437.

5. Tan E, Russell KP (2017) Surgical plume and its implications: A review of the risk and barriers to a safe work place. J Perioper Nurs Aust 30: 33-39.
6. Pavan N, Crestani A, Abrate A, Nunzio C, Esperto F, et al. (2020) Risk of virus contamination through surgical smoke during minimally invasive surgery: A systematic review of literature on a neglected issue revived in the covid-19 pandemic era. Eur Urol Focus 6: 1058-1069.

7. Liu Y, Song Y, Hu X, Yan L, Zhu X (2019) Awareness of surgical smoke hazards and enhancement of surgical smoke prevention among the gynecologists. J Cancer 10: 2788-2799.

8. Okoshi K, Tomizawa Y, Kinoshita K, Kobayashi K, Hasegawa S, et al. (2015) Health risks associated with exposure to surgical smoke for surgeons and operation room personnel. Surg Today 45: 957-965.

9. Rioux M, Garland A, Webster D, Reardon E (2013) HPV positive tonsillar cancer in two laser surgeons: Case reports. J Otolaryngol - Head Neck Surg 42: 2-5.

10. Allen JG, Ibrahim AM (2021) Indoor air changes and potential implications for SARS-CoV-2 Transmission. JAMA 325: 2112-2113. 